\title{
Lifestyle and environmental contributions to ovulatory dysfunction in women of polycystic ovary syndrome
}

Bingqian Zhang ${ }^{1,2,3,4}$, Wei Zhou ${ }^{1,2,3,4}$, Yuhua Shi ${ }^{1,2,3,4}$, Jun Zhang ${ }^{5}$, Linlin Cui ${ }^{1,2,3,4^{*}}$ and Zi-Jiang Chen ${ }^{1,2,3,4,6,7}$

\begin{abstract}
Background: Polycystic ovary syndrome (PCOS) is the most common reason of anovulatory infertility. Environmental factor is one of the main causes of PCOS, but its contribution to ovulatory dysfunction in PCOS remains unknown.

Methods: A total of 2217 infertile women diagnosed as PCOS according to Rotterdam criteria were recruited, including 1979 women with oligo-anovulation (OA group) and 238 women with normal -anovulation (non OA group). Besides, 279 healthy control women of reproductive age were enrolled as controls.

Results: Frequencies of snoring (PCOS-OA group, PCOS-non-OA group, control group: $29.30 \%$ vs $18.10 \%$ vs $11.50 \%$, $P<0.01$ ), smoking $(37.70 \%$ vs $28.10 \%$ vs $12.20 \%, P<0.01)$, plastic tableware usage $(38.30 \%$ vs $28.10 \%$ vs $25.40 \%, P<$ 0.01 ) and indoor decoration ( $32.10 \%$ vs $24.80 \%$ vs $16.80 \%, P<0.01$ ) were highest in PCOS-OA group. After adjusted for multivariable, difference remained significant between PCOS-OA group and the other two groups. PCOS-OA women preferred a meat favorable diet compared to PCOS-non-OA group $(54.60 \%$ vs $41.30 \%, P<0.01)$. There was no difference between three groups in exercise, frequency of insomnia, and alcohol consumption.
\end{abstract}

Conclusions: Smoking, snoring, hyper-caloric diet, plastic tableware usage and indoor decoration were found to be associated with an increased risk for ovulatory dysfunction in women suffering from PCOS.

Keywords: PCOS, Environmental factors, Lifestyle, Ovulatory dysfunction

\section{Background}

Polycystic ovary syndrome (PCOS) is a heterogeneous endocrinopathy with a prevalence of approximately $5.5-$ $19.9 \%$ [1]. It is characterized by irregular cycles, clinical or biochemical hyperandrogenism, and polycystic ovarian morphology (PCOM). Moreover, it is the most common cause of chronic anovulatory infertility [2]. As reported previously, $90.30-95.28 \%$ of PCOS women diagnosed by Rotterdam criteria were characterized with ovulatory dysfunction [3, 4]. Additionally, oligo-/anovulatory (OA) subjects showed more severe hormonal and metabolic derangements compared to PCOS women with normal

\footnotetext{
* Correspondence: fdclear3@126.com

${ }^{1}$ Center for Reproductive Medicine, Shandong University, No.157 of Jingliu Street, Jinan 250012, Shandong, China

${ }^{2}$ National Research Center for Assisted Reproductive Technology and Reproductive Genetics, Shandong University, No.44 of Wenhua Street, Jinan 250012, Shandong, China

Full list of author information is available at the end of the article
}

ovulation [4,5]. According to Chinese diagnostic criteria, ovulatory dysfunction was even considered as necessity for the diagnosis of the syndrome [6]. However, the pathogenesis has not been fully understood.

Environmental factors and genetic variants are both well accepted as main etiologic factors of PCOS. Several susceptibility genes have been reported via candidate gene screening and GWAS [7-9]. However, environmental components, being another key factor in the pathophysiology, have not been well documented. It was shown that environmental endocrine disruptors (EEDs) could perturb the hormonal regulation of the hypothalamic pituitary ovarian axis. Hence, EEDs are supposed to act as steroid-agonists and/or antagonists [10-12]. Previous studies also indicated a correlation between PCOS and contact history of EEDs, as well as changes of environment and lifestyle [13, 14]. However, the exact

(C) The Author(s). 2020 Open Access This article is distributed under the terms of the Creative Commons Attribution 4.0 International License (http://creativecommons.org/licenses/by/4.0/), which permits unrestricted use, distribution, and reproduction in any medium, provided you give appropriate credit to the original author(s) and the source, provide a link to the Creative Commons license, and indicate if changes were made. The Creative Commons Public Domain Dedication waiver (http://creativecommons.org/publicdomain/zero/1.0/) applies to the data made available in this article, unless otherwise stated. 
effect of environmental factors on ovulatory dysfunction in PCOS women remains unclear.

In our study, lifestyles and EED exposure of PCOS women with and without OA were analyzed in order to determine the association between environmental factors and ovulatory dysfunction of PCOS.

\section{Methods}

\section{Participants}

A total of 2217 PCOS women and 279 non-PCOS women were recruited from April 2006 to December 2009 in the Center for Reproductive Medicine, Shandong Provincial Hospital Affiliated to Shandong University. Among PCOS patients, patients with oligo-/anovulation were included in PCOS-OA group $(N=1979)$. Patients with normal menses but with hyperandrogenism and PCOM were included in the PCOS-non-OA group $(N=238)$. All of the participants were recruited from a prospective cohort study including women undergoing assisted reproductive technology treatment. The non-PCOS women who have previously participated in another studies were recruited as healthy controls $[15,16]$.

All PCOS women were diagnosed according to Rotterdam criteria with any two of following phenotypes: 1) OA, 2) hyperandrogenism, 3) PCOM [17, 18]. Moreover, absence of other causes of ovulatory dysfunction and hyperandrogenism including 21-hydroxylase deficiency, congenital adrenal hyperplasias, androgen-secreting tumors, Cushing's syndrome, hyperprolactinemia, and thyroid disease were excluded. OA was defined as the duration of menstrual cycle more than 35 days in length or a history of less than 8 spontaneous hemorrhagic episodes per year [19]. Hyperandrogenism was determined when there was either biochemical hyperandrogenemia (total testosterone levels in early follicular phase $\geq 60 \mathrm{ng} / \mathrm{dl}$ ) or hirsutism (modified Ferriman-Gallwey score $\geq 6$ ). Trans-vaginal ultrasonic examination was performed, and PCOM was considered when 12 or more follicles with a diameter of $2-9 \mathrm{~mm}$ were found in at least one ovary and/or the ovarian volume was over $10 \mathrm{ml}[17,18]$. All women in control group were undergoing treatment for tubal obstruction or male factor induced infertility. All of them had normal menstrual cycles.

\section{Clinical and biochemical measurements}

Information on age, height, weight, and medical history were recorded during clinical examination. Menstruation information of women in PCOS non-OA group were recorded without drug usage. Body mass index (BMI) was calculated as weight $(\mathrm{kg}) /$ height $\left(\mathrm{m}^{2}\right)$. Questionnaire including social information, life style and environmental contact history in daily life were completed by the same trained interviewer. Lifestyle referred to insomnia, snoring, meat favorable diet, smoking, alcohol consumption, tea drinking, and exercise duration. Assessment of environmental exposure includedthe usage of plastic tableware, indoor decoration, air freshener, and cooking oil fume. Definitions of those items were presented in Table 2.

Fasting blood sampling was collected during early follicular phase. The parameters including follicular stimulating hormone (FSH), luteinizing hormone ( $\mathrm{LH})$, total testosterone (TT), and prolactin (PRL) using chemiluminescence immunization. The intra- and inter-assay variation coefficients of variation are $<10 \%$.

\section{Statistical analysis}

Statistical analysis was performed using Statistical Package for the Social Sciences for Windows (version 22.0; SPSS Inc., Chicago, IL, USA). Normality of data was assessed by Q-Q plot. Continuous normal distributed variables were presented as mean $\pm \mathrm{SD}$. One-way ANOVA test and test were undertaken for continuous normal distributed variables. $\mathrm{X}^{2}$ analysis were undertaken for categorical variables. Multivariate logistic regression was performed to adjust potential confounders, such as age and BMI. Statistical significance was set at level of 0.05 .

\section{Results}

Basic characters were shown in Table 1. PCOS women were older (PCOS-OA group, PCOS-non-OA group, Control group: $31.11 \pm 3.71$ vs $31.56 \pm 3.49$ vs $29.81 \pm$ 3.77 yrs., $\mathrm{P}<0.01)$ and had a higher BMI $(24.90 \pm 4.09$ vs $25.31 \pm 4.39$ vs $22.93 \pm 3.86 \mathrm{~kg} / \mathrm{m} 2, \mathrm{P}<0.01)$ compared to controls. Results of univariate analysis were shown in Table 2. Frequency of snoring $(29.30 \%$ vs $18.10 \%$ vs $11.50 \%, P<0.01)$ and smoking $(37.70 \%$ vs $28.10 \%$ vs $12.20 \%, P<0.01)$ were different among three groups and were highest in the PCOS-OA group (Table 2, Fig. 1). After multivariate adjustment of age and BMI, these differences remained statistically significant except for the comparison of snoring between PCOS-non-OA and control group (Table 3). PCOS women, both with OA and

Table 1 Basic characteristic in oligo-/anovulation PCOS women, eumenorrheic PCOS women and control women

\begin{tabular}{lllll}
\hline & PCOS-OA & PCOS-non-OA & Con & $P$ \\
\hline No. & 1979 & 238 & 279 & \\
Age (yrs) & $31.11 \pm 3.71$ & $31.56 \pm 3.49$ & $29.81 \pm 3.77$ & $<0.01^{\text {a }}$ \\
BMl (kg/m2) & $24.90 \pm 4.09$ & $25.31 \pm 4.39$ & $22.93 \pm 3.86$ & $<0.01^{\text {a }}$ \\
Total AFC & $27.63 \pm 8.06$ & $27.68 \pm 9.23$ & $14.72 \pm 5.44$ & $<0.01^{\text {a }}$ \\
LH/FSH & $1.50 \pm 0.81$ & $1.58 \pm 0.85$ & $0.79 \pm 0.30$ & $<0.01^{a}$ \\
T (ng/dl) & $80.14 \pm 18.82$ & $67.79 \pm 23.46$ & $23.78 \pm 12.83$ & $<0.01^{a}$ \\
E2(pg/ml) & $45.73 \pm 19.36$ & $45.22 \pm 19.13$ & $34.87 \pm 25.42$ & $<0.01^{a}$
\end{tabular}

Data was presented as Mean \pm SD for normality distributions. One-way anova and $t$ test were used in continuous variables

Abbreviation: BMI Body mass index

${ }^{\mathrm{a}}$ significance was set at level of 0.05 
Table 2 Univariate Comparison in oligo-/anovulation PCOS women, eumenorrheic PCOS women and control women

\begin{tabular}{|c|c|c|c|c|}
\hline & PCOS-OA & PCOS-non-OA & Control & $P$ \\
\hline No. & 1979 & 238 & 279 & \\
\hline Meat favorable diet \%(N) & $54.60(1061)$ & 41.30(95) & NA & $<0.01^{a}$ \\
\hline Exercise duration per week \%(N) & & & & 0.08 \\
\hline Less than $10 \mathrm{~h}$ & $64.90(1274)$ & $57.10(133)$ & $65.60(181)$ & \\
\hline 10-20 h (including $10 \mathrm{~h}$ ) & $23.80(468)$ & $26.60(62)$ & $24.60(68)$ & \\
\hline More than $20 \mathrm{~h}$ (including $20 \mathrm{~h}$ ) & $11.30(221)$ & 16.30(38) & $9.80(27)$ & \\
\hline Insomnia\%(N) & & & & 0.18 \\
\hline Rare & $81.40(1596)$ & $87.60(205)$ & $83.10(231)$ & \\
\hline 1-3times/month & $11.00(215)$ & $6.40(15)$ & $9.40(26)$ & \\
\hline Every week & $7.60(149)$ & $6.00(14)$ & $7.60(21)$ & \\
\hline Snoring \%(N) & 29.30(579) & 18.10(43) & $11.50(32)$ & $<0.01^{\mathrm{a}}$ \\
\hline Smoking \%(N) & $37.70(734)$ & $28.10(66)$ & $12.20(34)$ & $<0.01^{a}$ \\
\hline Alcohol consumption \%(N) & $7.10(141)$ & $6.70(16)$ & $6.50(18)$ & 0.92 \\
\hline Tea drinking $(\%(\mathrm{~N})$ & $14.10(273)$ & $15.80(37)$ & $7.00(19)$ & $<0.01^{a}$ \\
\hline Plastic tableware usage $\%(\mathrm{~N})$ & $38.30(750)$ & $28.10(66)$ & $25.40(71)$ & $<0.01^{\mathrm{a}}$ \\
\hline Indoor decoration at home or workplace \%(N) & $32.10(635)$ & 24.80(59) & 16.80(47) & $<0.01^{\mathrm{a}}$ \\
\hline Air freshener usage $\%(\mathrm{~N})$ & 15.60(307) & $9.30(22)$ & $12.90(36)$ & $0.02^{\mathrm{a}}$ \\
\hline Cooking oil fume contact \%(N) & $53.00(1026)$ & 43.00(101) & $32.30(90)$ & $<0.01^{a}$ \\
\hline
\end{tabular}

Data was presented as Mean \pm SD and median (interquartile range) for normality and non-normality distributions respectively. One-way anova was used in continuous variables, while Chi square test was used in categorical variables

Smoke: Active smoking was defined as a person who currently smoke at least 1 cigarette per 3 days. Passive smoking was defined as a person who inhale smoke constantly from others in work or living area

Alcohol drinking: once a week for at least 6 months; Tea drinking: one cup per day for at least 6 months; Plastic tableware usage: 1 time per day for at least 6 months; Indoor decoration at home or workplace: indoor painting performed or large furniture moved within two years before incidence of dysmenorrhea ; Air freshener usage: 3 times per week for at least 6 months; Cooking oil fume contact: Cooking oil fume contact was defined as women who cooked at home or workplace at least once per 3 days

${ }^{a}$ significance was set at level of 0.05
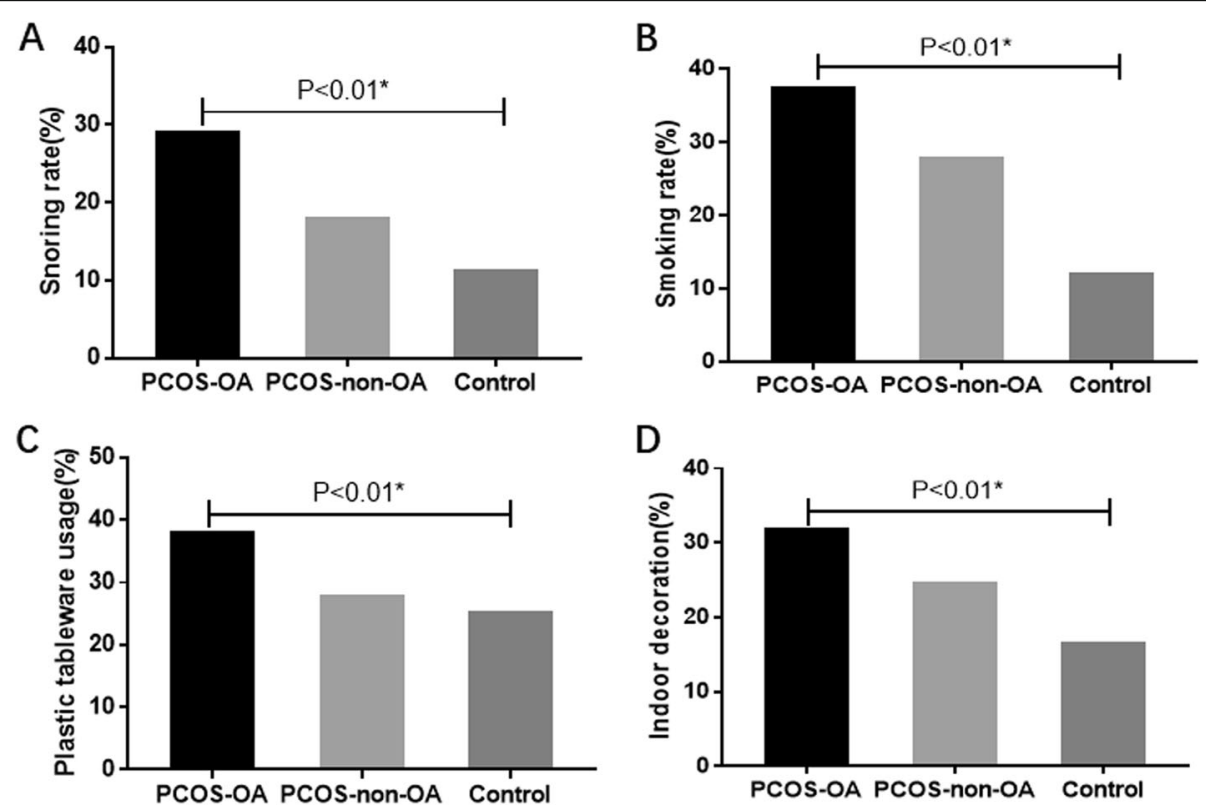

Fig. 1 Lifestyle and environmental presence in oligo-/anovulation PCOS, eumenorrheic PCOS and control women. a Presence of snoring $\mathbf{b}$ Presence of smoking $\mathbf{c}$ Presence of plastic tableware usage $\mathbf{d}$ Presence of indoor decoration. OA:oligo-/anovulation. *: significance was set at level of $0.017(0.05 / 3)$. 
Table 3 Multivariate Comparison in oligo-/anovulation PCOS women, eumenorrheic PCOS women and control women

\begin{tabular}{|c|c|c|c|c|c|c|}
\hline & \multicolumn{2}{|c|}{ PCOS-OA vs PCOS-non-OA } & \multicolumn{2}{|c|}{ PCOS-OA vs Control } & \multicolumn{2}{|c|}{ PCOS-non-OA vs Control } \\
\hline & $\mathrm{OR}(95 \% \mathrm{Cl})$ & $P$ & $\mathrm{OR}(95 \% \mathrm{Cl})$ & $P$ & $\mathrm{OR}(95 \% \mathrm{Cl})$ & $P$ \\
\hline Meat favorable diet $\%(\mathrm{~N})^{\text {a }}$ & $1.69(1.28,2.23)$ & $<0.01^{\mathrm{b}}$ & NA & NA & NA & NA \\
\hline Snoring $\%(N)^{a}$ & $2.17(1.51,3.12)$ & $<0.01^{\mathrm{b}}$ & $2.25(1.49,3.40)$ & $<0.01^{\mathrm{b}}$ & $0.96(0.56,1.65)$ & 0.89 \\
\hline Smoking $\%(N)^{a}$ & $1.52(1.13,2.05)$ & $<0.01^{\mathrm{b}}$ & $3.69(2.47,5.52)$ & $<0.01^{\mathrm{b}}$ & $2.39(1.47,3.89)$ & $<0.01^{\mathrm{b}}$ \\
\hline Tea drinking $\%(\mathrm{~N})^{a}$ & $0.90(0.62,1.31)$ & 0.58 & $1.88(1.12,3.14)$ & $0.02^{b}$ & $2.11(1.14,3.89)$ & $0.02^{\mathrm{b}}$ \\
\hline Plastic tableware usage $\%(\mathrm{~N})^{\text {a }}$ & $1.55(1.14,2.09)$ & $<0.01^{\mathrm{b}}$ & $2.00(1.47,2.72)$ & $<0.01^{\mathrm{b}}$ & $1.31(0.87,1.98)$ & 0.19 \\
\hline Indoor decoration at home or workplace $\%(\mathrm{~N})^{a}$ & $1.40(1.03,1.91)$ & $0.03^{b}$ & $2.53(1.78,3.57)$ & $<0.01^{\mathrm{b}}$ & $1.86(1.19,2.92)$ & $<0.01^{\mathrm{b}}$ \\
\hline Air freshener usage $\%(N)^{a}$ & $1.77(1.12,2.79)$ & $0.01^{\mathrm{b}}$ & $1.27(0.86,1.88)$ & 0.23 & $0.69(0.38,1.24)$ & 0.21 \\
\hline Cooking oil fume contact $\%(\mathrm{~N})^{\text {a }}$ & $1.48(1.13,1.95)$ & $<0.01^{\mathrm{b}}$ & $1.10(0.84,1.46)$ & 0.49 & $0.73(0.50,1.06)$ & 0.10 \\
\hline
\end{tabular}

Abbreviation: BMI Body mass index

${ }^{a}$ Multivariate logistic regression was performed for categorical variables. Adjusted parameters included age, BMl, and groups

${ }^{\mathrm{b}}$ significance was set at level of 0.05

without OA, preferred to drink tea more often than control women, even after adjustment (Table 3). Diet composition was only recorded in PCOS women. The comparison showed that PCOS-OA women had higher rate of meat favorable diet than their non-OA counterparts $(54.60 \%$ vs $41.30 \%, P<0.01 ; 1.69(1.28,2.23), P<$ 0.01 after adjustment). No differences were found in exercise duration $(P=0.08)$, frequency of insomnia $(P=$ $0.18)$, alcohol consumption $(P=0.92)$ amongst the three groups.

The higher usage of plastic tableware $(38.30 \%$ vs $28.10 \%$ vs $25.40 \%, P<0.01)$ and indoor decoration (32.10\% vs $24.80 \%$ vs $16.80 \%, P<0.01)$ were found in PCOS-OA women compared with either PCOS-non-OA or control women (Table 2, Fig. 1). Similar trends were found after adjusting age and BMI. Furthermore, indoor decoration showed a gradient trend among three groups [OR: $1.40,95 \% \mathrm{CI}: 1.03-1.91, P=0.03$ for PCOS-OA vs PCOS-non-OA, OR: $2.53, .95 \% \mathrm{CI}: 1.78$ to 3.57$), P<0.01$ for PCOS-OA vs Control, OR: 1.86, 95\%CI: 1.19 to 2.92, $P<0.01$ PCOS-non-OA vs control, Table 3]. Air refresher usage $(15.60 \%$ vs $9.30 \%$ vs $12.90 \%, P<0.01)$, and cooking oil fume contact $(53.00 \%$ vs $43.00 \%$ vs $32.30 \%$, $P<0.01)$ were more frequent in PCOS-OA women compared to their non-OA counterparts. But these differences were non-significant after adjustment (Table 3). We also compared education, occupation, sleep duration, source of drinking water, pesticide free of fruits and vegetables, and microwave usage among three groups, but found no difference (data not shown).

\section{Discussion}

In the present study, smoking, snoring, meat favorable diet, and usage of plastic tableware and indoor decoration were found to be associated with oligo or anovulation in PCOS women. Moreover, smoking and indoor decoration contact seemed to have a dose-dependent effect. Tea drinking was positively associated with PCOS but not ovulatory dysfunction. These factors indicated that lifestyle and environmental endocrine disruptors may associated with the pathophysiology of PCOS.

Smoking was found to be associated with ovulatory dysfunction in a dose-dependent way in our study. Several studies have observed that smoke toxicants can disrupt folliculogenesis, leading to premature luteinization of preovulatory follicle. These toxicants can also decrease oocytes maturation and ultimately accelerated depletion of the primordial follicle pool $[20,21]$. However, other studies found there was no correlation between smoking and OA in PCOS women [22, 23]. The main difference between previous studies and our study is the definition of smoking. In our study, exposure to smoking included both active and passive smoking, whereas other studies only included active smoking.

Snoring was found to be another lifestyle indicator associated with OA in PCOS. It is one of the earliest symptoms of obstructive sleep apnea (OSA), which is a complex disorder characterized by repetitive collapse of the pharyngeal airway during sleep [24]. Previous studies have reported that PCOS is associated with a reduction in REM sleep stage time and increased risk for OSA [25-27]. Alerted reproductive hormone secretion (i.e. high androgen and low estrogen levels) might contribute to the higher prevalence of OSA [28]. Furthermore, it was indicated that low estradiol-to-testosterone ratio was associated with chronic oligo-anovulatory cycles in PCOS [29].

The present study also confirmed the correlation of meat favorable diet and the risk of ovulatory dysfunction, which was consisted with findings in animal studies. Previous studies showed that a decrease of primordial and Graafian follicles in high-fat feeding rat [30]. Insulin resistance (IR) was supposed as one of the potential mechanisms. Studies in both animal and human beings demonstrated that hypercaloric diet will induce IR and $\beta$-cell dysfunction [31, 32]. IR was found to interrupt follicle development through 
inhibiting hypothalamic positive feedback to estradiol (E2). Besides, the direct lesion on ovulation through kisspeptin down-expression and granulosa cell apoptosis were also indicated as underlying mechanism in high-fat diet feeding mice [33, 34].

Nowadays, more and more people pay attention to the impact of EEDs on female reproductive health. We found plastic tableware usage and indoor decoration were associated with PCOS ovulatory dysfunction in the present study. It was demonstrated that Bisphenol A (BPA), the main component of plastic containers, had a positive association with endocrine disturbances in PCOS [35, 36]. It acted as a potent binder of sex hormone-binding globulin [37] and had a bidirectional interaction effect with androgens [38, 39], which may result in perturbed ovarian steroidogenesis and folliculogenesis. However, recent studies indicated that the BPA exposure didn't alter ovulation in mice [40, 41]. Hence, more in vivo studies were needed to replicate the association of BPA exposure and ovulatory dysfunction, and to elucidate the mechanism. Except for BPA, the plasticizer and its substitute diisononyl phthalate (DiNP), were other EED's found in many consumers. DEHP or DiNP exposure was demonstrated to accelerate primordial follicle recruitment by up-regulating the PI3K pathway, and lead to prolonged estrous cyclicity and subfertility in female mouse. The pathological effect could last even long after cessation of exposure $[42,43]$.

We also found a positive association between indoor decoration and PCOS ovulatory dysfunction. However, there were limited studies focusing on the association between indoor decoration and PCOS [13]. It was found that organic solvents, the most important constituents for indoor decoration, had a negative influence on glucose metabolism impairment $[44,45]$. Hundreds of chemicals were detected in organic solvents that could activate tumor necrosis factor $\alpha$ (TNF $\alpha$ ), one of the most famous proinflammatory cytokines, which resulted excessive hepatic glucose formation, inhibited muscular glucose uptake, and impaired insulin sensitivity $[46,47]$ through different pathways [47-49]. There was a definite association between insulin resistance and interrupted follicle development. This may explain the correlation between indoor decoration and PCOS.

The strength of the present study was that it has large samples. Besides, it evaluated the association of lifestyle and exposure to environmental pollutants with ovulatory dysfunction in PCOS women systemically, which would provide important indication on next-step etiological study of impacts of environment factors on ovulation dysfunction and PCOS. However, it still had several limitations. Firstly, as a self-report questionnaire, social desirability cannot be eliminated. Secondly, only a minority of Chinese women smoke due to cultural habits. Thus, the sample size is too small to conduct further subgroup analysis to examine the contribution of active or passive smoking. Besides, the exact caloric intake per day was not recorded in our data. Comparison of diet composition only cannot provide further does-dependent evidence. Specific design studies involving caloric intake were needed to explore the effect of diet on oligo-/anovulatory in PCOS.

\section{Conclusions}

Ovulatory dysfunction in PCOS is related to unhealthy lifestyle and environmental pollutants exposure. Hence, lifestyle modification as the first-line therapy for PCOS especially women with OA should be promoted more vigorously.

\section{Abbreviations}

BMI: Body mass index; BPA: Bisphenol A; DEHP: Di(2-ethylhexyl) phthalate; DiNP: Diisononyl phthalate; EEDs: Environmental endocrine disruptors; FSH: Follicular stimulating hormone; LH: Luteinizing hormone; OA: Oligoanovulation; PCOM: Polycystic ovarian morphology; PCOS: Polycystic ovary syndrome; PRL: Prolactin; TNFa: Tumor necrosis factor $a ;$; T: Total testosterone

\section{Acknowledgments}

We are grateful to Jiangtao Zhang for data collecting. We especially thank all women participating in this study.

\section{Authors' contributions}

ZJC oversaw the entire project and revised the manuscript. LC and ZJC contributed to the conception and design of the study. BZ analyzed the data and drafted the article. WZ, YS and JZ contributed to the acquisition of samples and revised the article. All authors gave their final approval of the version to be published.

\section{Funding}

This research was supported by the National Key Research and Development Program of China (2016YFC10002) and the Shandong Provincial Key Research and Development Program (2017G006036, 2018YFJH0504). The funding body had no role in study design, data collection, analysis, interpretation of data and preparation the manuscript.

\section{Availability of data and materials}

The datasets are available from the corresponding author on reasonable request.

\section{Ethics approval and consent to participate}

The present study was approved by the Local Medical Institutional Review Board of the Reproductive Medicine of Shandong University. Written

informed consent was obtained from each participant.

\section{Consent for publication}

Not applicable.

\section{Competing interests}

The authors declare that they have no competing interests.

\section{Author details}

${ }^{1}$ Center for Reproductive Medicine, Shandong University, No.157 of Jingliu Street, Jinan 250012, Shandong, China. ${ }^{2}$ National Research Center for Assisted Reproductive Technology and Reproductive Genetics, Shandong University, No.44 of Wenhua Street, Jinan 250012, Shandong, China. ${ }^{3}$ Key laboratory of Reproductive Endocrinology of Ministry of Education, Shandong University, No.44 of Wenhua street, Jinan 250012, Shandong, China. ${ }^{4}$ Shandong Provincial Clinical Medicine Research Center for Reproductive Health, Shandong University, No.157 of Jingliu Street, Jinan 250012, Shandong, China. ${ }^{5}$ Ministry of Education-Shanghai Key Laboratory of Children's 
Environmental Health, Xinhua Hospital, School of Medicine, Shanghai Jiao-Tong University, No.1665 of Kongjiang Street, Shanghai 200092, China. ${ }^{6}$ Shanghai Key Laboratory for Assisted Reproduction and Reproductive Genetics, No.845 of Lingshan Street, Shanghai 200088, China. ${ }^{7}$ Center for Reproductive Medicine, Ren Ji Hospital, School of Medicine, Shanghai Jiao Tong University, No.845 of Lingshan Street, Shanghai 200088, China.

\section{Received: 12 November 2019 Accepted: 20 January 2020}

\section{Published online: 30 January 2020}

\section{References}

1. Azziz R, Carmina E, Chen Z, Dunaif A, Laven JS, Legro RS, Lizneva D, Natterson-Horowtiz B, Teede HJ, Yildiz BO. Polycystic ovary syndrome. Nat Rev Dis Primers. 2016;2:16057.

2. Bani Mohammad M, Majdi Seghinsara A. Polycystic ovary syndrome (PCOS), diagnostic criteria, and AMH. Asian Pac J Cancer Prev. 2017;18(1):17-21.

3. Xu X, Shi Y, Cui Y, Ma J, Che L, Chen ZJ. Endocrine and metabolic characteristics of polycystic ovary syndrome in Chinese women with different phenotypes. Clin Endocrinol. 2012;76(3):425-30.

4. Panidis D, Tziomalos K, Misichronis G, Papadakis E, Betsas G, Katsikis I, Macut $D$. Insulin resistance and endocrine characteristics of the different phenotypes of polycystic ovary syndrome: a prospective study. Hum Reprod. 2012;27(2):541-9.

5. Mumford SL, Schisterman EF, Siega-Riz AM, Gaskins AJ, Steiner AZ, Daniels J, Olshan AF, Hediger ML, Hovey K, Wactawski-Wende J, et al. Cholesterol, endocrine and metabolic disturbances in sporadic anovulatory women with regular menstruation. Hum Reprod. 2011;26(2):423-30.

6. Reproductive endocrinology group of Chinese medical association. The diagnosis and treatment of polycystic ovary syndrome in China. Chin J Obstet Gynecol. 2018;53(1).

7. Chen ZJ, Zhao H, He L, Shi Y, Qin Y, Shi Y, Li Z, You L, Zhao J, Liu J, et al. Genome-wide association study identifies susceptibility loci for polycystic ovary syndrome on chromosome 2p16.3, 2p21 and 9q33.3. Nat Genet. 2011; 43(1):55-9.

8. Shi Y, Zhao H, Shi Y, Cao Y, Yang D, Li Z, Zhang B, Liang X, Li T, Chen J, et al. Genome-wide association study identifies eight new risk loci for polycystic ovary syndrome. Nat Genet. 2012;44(9):1020-5.

9. Cui L, Li G, Zhong W, Bian Y, Su S, Sheng Y, Shi Y, Wei D, Zhang W, Zhao H, et al. Polycystic ovary syndrome susceptibility single nucleotide polymorphisms in women with a single PCOS clinical feature. Hum Reprod. 2015:30(3):732-6.

10. Speranza A, Crosti P, Malerba M, Stocchi O, Scoccianti $V$. The environmental endocrine disruptor, bisphenol a, affects germination, elicits stress response and alters steroid hormone production in kiwifruit pollen. Plant Biol (Stuttg). 2011;13(1):209-17.

11. Belcher SM, Chen Y, Yan S, Wang HS. Rapid estrogen receptor-mediated mechanisms determine the sexually dimorphic sensitivity of ventricular myocytes to 17 beta-estradiol and the environmental endocrine disruptor bisphenol a. Endocrinology. 2012;153(2):712-20.

12. Rattan S, Zhou C, Chiang C, Mahalingam S, Brehm E, Flaws JA. Exposure to endocrine disruptors during adulthood: consequences for female fertility. J Endocrinol. 2017;233(3):R109-r129.

13. Huang WJ, Liu JY, Li LN. Analysis of environmental factors and polycystic ovary syndrome. Zhonghua Fu Chan Ke Za Zhi. 2007;42(5):302-4.

14. Zhang J, Liu XF, Liu Y, Xu LZ, Zhou LL, Tang LL, Zhuang J, Li TT, Guo WQ, $\mathrm{Hu} R$, et al. Environmental risk factors for women with polycystic ovary syndrome in China: a population-based case-control study. J Biol Regul Homeost Agents. 2014;28(2):203-11.

15. Wang W, Zhou W, Wu S, Liang F, Li Y, Zhang J, Cui L, Feng Y, Wang Y. Perfluoroalkyl substances exposure and risk of polycystic ovarian syndrome related infertility in Chinese women. Environ Pollut. 2019;247:824-31.

16. Zhou W, Fang F, Zhu W, Chen ZJ, Du Y, Zhang J. Bisphenol A and Ovarian Reserve among Infertile Women with Polycystic Ovarian Syndrome. Int J Environ Res Public Health. 2016;(1):14. https://doi.org/10.3390/ ijerph14010018.

17. Rotterdam ESHRE/ASRM-Sponsored PCOS Consensus Workshop Group. Revised 2003 Consensus on diagnostic criteria and long-term health risks related to polycystic ovary syndrome. Fertil Steril. 2004;81(1):19-25.

18. Rotterdam ESHRE/ASRM-Sponsored PCOS consensus workshop group. Revised 2003 Consensus on diagnostic criteria and long-term health risks related to polycystic ovary syndrome (PCOS). Hum Reprod. 2004;19(1):41-7.
19. Azziz R, Woods KS, Reyna R, Key TJ, Knochenhauer ES, Yildiz BO. The prevalence and features of the polycystic ovary syndrome in an unselected population. J Clin Endocrinol Metab. 2004;89(6):2745-9.

20. Sadeu JC, Foster WG. Cigarette smoke condensate exposure delays follicular development and function in a stage-dependent manner. Fertil Steril. 2011; 95(7):2410-7.

21. Dechanet C, Anahory T, Mathieu Daude JC, Quantin X, Reyftmann L, Hamamah S, Hedon B, Dechaud H. Effects of cigarette smoking on reproduction. Hum Reprod Update. 2011;17(1):76-95.

22. Cupisti S, Haberle L, Dittrich R, Oppelt PG, Reissmann C, Kronawitter D, Beckmann MW, Mueller A. Smoking is associated with increased free testosterone and fasting insulin levels in women with polycystic ovary syndrome, resulting in aggravated insulin resistance. Fertil Steril. 2010;94(2):673-7.

23. Legro RS, Chen G, Kunselman AR, Schlaff WD, Diamond MP, Coutifaris C, Carson SA, Steinkampf MP, Carr BR, McGovern PG, et al. Smoking in infertile women with polycystic ovary syndrome: baseline validation of self-report and effects on phenotype. Hum Reprod. 2014;29(12):2680-6.

24. Epstein LJ, Kristo D, Strollo PJ Jr, Friedman N, Malhotra A, Patil SP, Ramar K, Rogers R, Schwab RJ, Weaver EM, et al. Clinical guideline for the evaluation, management and long-term care of obstructive sleep apnea in adults. J Clin Sleep Med. 2009;5(3):263-76.

25. Hachul H, Polesel DN, Tock L, Carneiro G, Pereira AZ, Zanella MT, Tufik S, Togeiro SM. Sleep disorders in polycystic ovary syndrome: influence of obesity and hyperandrogenism. Rev Assoc Med Bras. 2019;65(3):375-83.

26. Tasali E, Van Cauter E, Ehrmann DA. Relationships between sleep disordered breathing and glucose metabolism in polycystic ovary syndrome. J Clin Endocrinol Metab. 2006;91(1):36-42.

27. Kahal H, Kyrou I, Uthman OA, Brown A, Johnson S, Wall PDH, Metcalfe A, Parr DG, Tahrani AA, Randeva HS. The prevalence of obstructive sleep apnoea in women with polycystic ovary syndrome: a systematic review and metaanalysis. Sleep Breath. 2019. https://doi.org/10.1007/s11325-019-01835-1.

28. Tasali E, Van Cauter E, Ehrmann DA. Polycystic ovary syndrome and obstructive sleep apnea. Sleep Med Clin. 2008;3(1):37-46.

29. Amato MC, Verghi M, Nucera M, Galluzzo A, Giordano C. Low estradiol-totestosterone ratio is associated with oligo-anovulatory cycles and atherogenic lipidic pattern in women with polycystic ovary syndrome. Gynecol Endocrinol. 2011;27(8):579-86.

30. Sohrabi M, Roushandeh AM, Alizadeh Z, Vahidinia A, Vahabian M, Hosseini M. Effect of a high fat diet on ovary morphology, in vitro development, in vitro fertilisation rate and oocyte quality in mice. Singap Med J. 2015;56(10):573-9.

31. Black MH, Watanabe RM, Trigo E, Takayanagi M, Lawrence JM, Buchanan TA, Xiang $\mathrm{AH}$. High-fat diet is associated with obesity-mediated insulin resistance and beta-cell dysfunction in Mexican Americans. J Nutr. 2013;143(4):479-85.

32. Mubarak A, Hodgson JM, Considine MJ, Croft KD, Matthews VB. Supplementation of a high-fat diet with chlorogenic acid is associated with insulin resistance and hepatic lipid accumulation in mice. J Agric Food Chem. 2013;61(18):4371-8.

33. Wu Y, Zhang Z, Liao $X$, Wang Z. High fat diet triggers cell cycle arrest and excessive apoptosis of granulosa cells during the follicular development. Biochem Biophys Res Commun. 2015;466(3):599-605.

34. Zhou Q, Chen H, Yang S, Li Y, Wang B, Chen Y, Wu X. High-fat diet decreases the expression of Kiss1 mRNA and kisspeptin in the ovary, and increases ovulatory dysfunction in postpubertal female rats. Reprod Biol Endocrinol. 2014;12:127.

35. Kandaraki E, Chatzigeorgiou A, Livadas S, Palioura E, Economou F, Koutsilieris M, Palimeri S, Panidis D, Diamanti-Kandarakis E. Endocrine disruptors and polycystic ovary syndrome (PCOS): elevated serum levels of bisphenol a in women with PCOS. J Clin Endocrinol Metab. 2011;96(3):E480-4.

36. Rutkowska A, Rachon D. Bisphenol a (BPA) and its potential role in the pathogenesis of the polycystic ovary syndrome (PCOS). Gynecol Endocrinol. 2014;30(4):260-5.

37. Dechaud H, Ravard C, Claustrat F, de la Perriere AB, Pugeat $M$. Xenoestrogen interaction with human sex hormone-binding globulin (hSHBG). Steroids. 1999:64(5):328-34.

38. Takeuchi T, Tsutsumi O, Ikezuki Y, Kamei Y, Osuga Y, Fujiwara T, Takai Y, Momoeda M, Yano T, Taketani Y. Elevated serum bisphenol a levels under hyperandrogenic conditions may be caused by decreased UDPglucuronosyltransferase activity. Endocr J. 2006;53(4):485-91.

39. Hanioka N, Jinno H, Nishimura T, Ando M. Suppression of male-specific cytochrome P450 isoforms by bisphenol a in rat liver. Arch Toxicol. 1998; 72(7):387-94. 
40. Moore-Ambriz TR, Acuna-Hernandez DG, Ramos-Robles B, Sanchez-Gutierrez M, Santacruz-Marquez R, Sierra-Santoyo A, Pina-Guzman B, Shibayama M, Hernandez-Ochoa I. Exposure to bisphenol a in young adult mice does not alter ovulation but does alter the fertilization ability of oocytes. Toxicol Appl Pharmacol. 2015;289(3):507-14.

41. Wang W, Hafner KS, Flaws JA. In utero bisphenol a exposure disrupts germ cell nest breakdown and reduces fertility with age in the mouse. Toxico Appl Pharmacol. 2014;276(2):157-64.

42. Chiang C, Flaws JA. Subchronic exposure to Di(2-ethylhexyl) phthalate and Diisononyl phthalate during adulthood has immediate and long-term reproductive consequences in female mice. Toxicol Sci. 2019;168(2):620-31.

43. Hannon PR, Peretz J, Flaws JA. Daily exposure to Di(2-ethylhexyl) phthalate alters estrous cyclicity and accelerates primordial follicle recruitment potentially via dysregulation of the phosphatidylinositol 3-kinase signaling pathway in adult mice. Biol Reprod. 2014:90(6):136.

44. Merker GH, Metzner G, Raabe F. $\mathrm{T}(\mathrm{H} 1)$-directed modulation of in vitro cytokine production in human peripheral blood mononuclear cells by styrene-7.8-oxide. Toxicol Lett. 2006;160(2):105-11.

45. Won YL, Ko Y, Heo KH, Ko KS, Lee MY, Kim KW. The effects of long-term, low-level exposure to monocyclic aromatic hydrocarbons on Worker's insulin resistance. Saf Health Work. 2011;2(4):365-74.

46. Ruan H, Lodish HF. Insulin resistance in adipose tissue: direct and indirect effects of tumor necrosis factor-alpha. Cytokine Growth Factor Rev. 2003; 14(5):447-55.

47. Karagozler AA, Mehmet N, Batcioglu K. Effects of long-term solvent exposure on blood cytokine levels and antioxidant enzyme activities in house painters. J Toxicol Environ Health A. 2002;65(17):1237-46.

48. Shapiro L, Scherer PE. The crystal structure of a complement-1q family protein suggests an evolutionary link to tumor necrosis factor. Curr Biol. 1998;8(6):335-8

49. Hotamisligil GS, Peraldi P, Budavari A, Ellis R, White MF, Spiegelman BM. IRS-1mediated inhibition of insulin receptor tyrosine kinase activity in TNF-alphaand obesity-induced insulin resistance. Science. 1996;271(5249):665-8.

\section{Publisher's Note}

Springer Nature remains neutral with regard to jurisdictional claims in published maps and institutional affiliations.

Ready to submit your research? Choose BMC and benefit from:

- fast, convenient online submission

- thorough peer review by experienced researchers in your field

- rapid publication on acceptance

- support for research data, including large and complex data types

- gold Open Access which fosters wider collaboration and increased citations

- maximum visibility for your research: over $100 \mathrm{M}$ website views per year

At $\mathrm{BMC}$, research is always in progress.

Learn more biomedcentral.com/submissions 